\title{
Applications of a-Si:H Radiation Detectors
}

I.Fujieda, G.Cho, M.Conti, J.Drewery, S.N.Kaplan, V.Perez-Mendez, S.Qureshi, Lawrence Berkeley Laboratory, Berkeley, CA.94720. and R.A.Street, Xerox Palo Alto Research Center, Palo Alto CA.94304.

\section{Abstract}

Device structures and operation prin iples are described for detecting various kinds of radiation with ydrogenated amorphous silicon (a-Si:H) layers. With some new configurations such as the buried $p-i-n$ structure and the use of interdigitated electrodes, the a$\mathrm{Si}: \mathrm{H}$ radiation detectors will find their applications in many fields of science. Some applications in high energy physics, medical imaging, materials sciences and life sciences are discussed in this paper.

\author{
LBL -27457
}

DE89 015230

\section{DISCLAIMER}

This report was prepured as an eccount of work sponsored by an acency of the United States Government. Neither the Uaited States Governmeal nor any asency thereof, nor any of their employees, makes any werranty, exprese or implied, or anumes any legal liability or reapona. bility for the accuracy, completeneas, or usefulness of any information, apparatus, product, or process discloed, or represents that its use would not infringe privately owned rights. Reforence herein to any specific commercial product, procean, or service by trade name, trademart, manufacturer, or ofherwixe does uot necesararily constitute or imply its endorsement, recommendation, or favoring by the United States Government or any asency thereof. The views and opinions of authors expreased herein do not neceasurily state or refloct those of the United States Government or any agancy thereor.

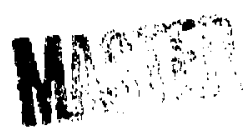




\section{Introduction}

Hydrogenated amorphous silicon $(\mathrm{a}-\mathrm{Si}: \mathrm{H})$ is now widely used in industry as the material for solar cells, facsimile heads and liquid crystal display drivers. The thickness of a-Si:H layers of these devices is less than $2 \mu \mathrm{m}$, which is enough to absorb all of the visible light as well as providing enough material for constructing thin film transistors (TFTs).

Early efforts to make radiation detectors with a-Si:H were limited to this thickness range $[1,2,3]$. Wei et al. [1] made a 16 element X-ray sensor with each element size of $3 \mathrm{~mm} \times 16 \mathrm{~mm}$. X-rily fluences were successfully detected by this device with a good linearity between the output signal size and the input $X$-raly intensity. When coupled to a phosphor such as $\mathrm{CdWO}_{4}$ and $\mathrm{ZnS}(\mathrm{Ni})$ ats in the case of $X$-ray films, a larger output was obtained due to the increased interaction efficiency of the individual $X$-ray photons. Mocliiki et al. [2] used a facsimile head to detect $X$-ray fluences and obtained similar results with an improved spatial resolution. Naruse et al. [3] have proposed the concept of the metal/a-Si:H multilayer structure, which looks like a miniature calorimeter as used in high energy physics experiments. While this structure has some potential as a single particle detector rather than a flux Jetector, the difficulty of the large detector capacitance remains unsolved. Since the a-Si:H layers in these devices are limited to a few $\mu \mathrm{m}$ thickness and their electrodes are all parallel plates, the detector capacitance is fairly large and so is the noise component known as the deita noise in the standard nuclear electronics for single particle detection [4]. Therefore, it is difficult to operate these devices in pulse mode (counting individual particles).

Our previous work $[5,6,7,8,9]$ focused on making thick a-Si:H layers up to $40 \mu \mathrm{m}$ with low detector capacitance $(10-100 \mathrm{pF})$ for single particle counting so that the incoming radiation deposits enough energy in the material to produce a large signal well above the noise. While some new production techniques of a-Si:H such as the VHF-GD $[10,11,12]$ and the Controlled Plasma Magnetron Method [13] are promising for fast deposition of a-Si:H layers with low ionizable defect density, they were not available to us and all of our thick samples were provided by Xerox and Glasstech Solar using the standard $13.54 \mathrm{MHz}$ Glow Discharge method at a deposition rate of about $1 \mu \mathrm{m} /$ hour. These thick layers can detect various kinds of radiation (individual charged particles, $\gamma$ rays and $X$-ray fluences). $A$ simple method to analyze charge collection in thick a-Si:H layers with 
light pulses of rarious wave lengths was devised 16] and the signal generation process was modeled [8].

In the next section, detection principles and structures of position sensitive a-Si:H radiation deteciors are reviewed. Some promising technologies in fabricating these a-Si:H detectors will be summarized. Various fields of application and devices utilizing al-Si:H detectors will be described in the following sections.

2. Delection principles..nd an sensitivity

Figure $\mathrm{I}$ summatizes the detection principles of a- $\mathrm{Si}: \mathrm{H}$ radiation delectors. In the direct detection scheme as shown in lïg.l(a), charged particles produce ionization events in the thick $(50-60 \mu \mathrm{m})$ depleted a-Si:H layer. The average energy required to produce one clectron-hole pair known as the $W$ value is about $6 \mathrm{eV}[5]$. This is larger than the gap energy of a-Sj·H (about $1.8 \mathrm{eV}$ ) because a part of the energy of incoming radiation is dissipated by the non-ionization events. These radiation-induced electron-hole pairs are drifted by the electric field, inducing charges on the electrodes which are amplified by the external electronics. In the indirect detection scheme shown in Fig.1(b), a phosphor converts the incident radiation (charged particles, $\gamma$ rays or $X$-ray fluences) into visible light $(\lambda=400$ $550 \mathrm{~nm}$ ) which is then absorbed mostly at the surface of the thin (1$2 \mu \mathrm{m})$ a-Si:H layer. Electrons and holes are created near the surface and are drifted by the field. Here, to take advantage of the higher electron mobility, the a-Si:H layer is biased so that electrons drift through the layer while holes are immediately collected by the nearby electrode. The readout electronics is designed to count either individual particles or fluences, depending on the applications of the detectors. In the calorimeter scheme as shown in Fig.l(c), a number of metal/a-Si:H layers are stacked together. The incident $X$-ray photons undergo photoelectric and Compton scattering interactions preferentially in the metal layers due to their higher atomic number. The resultant electrons which emerge from the metal converter layers produce electron-hole pairs in the a-Si:H layers. Here also, the readout electronics can be designed to count either individual particles or fluences, depending on the specific applications of the detectors.

One dimensional (1D) or two dimensional (2D) position sensitivity is achieved by dividing the electrode into narrow strips or small pixels and providing individual readout electronics for each strip or pixel. This is shown schematically in Fig.2. Up to now, only 
strip detectors with the current-mode readout electronics made from partly crystalline silicon and partly a-Si:H have been made[1,2]. Readout electronics for single particle detection is available in the form of crystalline silicon integrated circuit chips such as those designed at LBL for high energy physics experiments[14]. Taking advantage of this existing chip, single-particle-counting strip detectors can be built by wire-bonding each strip to one channel of the chip as shown in Fig.2(a). A strip or pixel device with the front end electronics made from a-Si:H or poly-Si TFTs on each strip or pixel as shown in Fig.2(b) and (c) has been proposed by us [7] and others [15]. In Fig.2(a), the electronics cell is mounted next to the detector strips. For fluence detection, the readout electronics are simple switching devices to address strips sequentially as in the case of facsimile heads. For individual particle or single $\gamma$ photon counting, the readout electronics contain a high-gain charge-sensitive amplifier stage and a sequential addressing stage as in the case of the LBL chip[14]. In Fig.2(c), vertical integration of the electronics and the addressing bus lines enables us to readout each pixel signal without reducing the sensitive area to radiation. Also shown is the schematic of the electronics on each pixel. Again, fluence detection needs a simple switching device to address $\mathrm{X}$ and $\mathrm{Y}$ coordinates while single particle counting requires a charge-sensitive amplifier stage in addition to the addressing stage. Design considerations for the readout electronics for single particle detection and noise measurements of the a-Si:H and poly-Si TFTs are currently under way and some results have been published $[9,16]$. While this electronics development needs a fair amount of effort, it can be shared with industry which may find a large market for a-Si:H radiation detectors such as described in this paper.

\section{New technologies}

There are three promising technologies which we are developing which can improve the detector performance for many applications.

\section{Buried $p-i-n$ structure}

For direct detection of high linear energy transfer (LET) particles such as $\alpha$ particles and heavy ions, a significant number of the radiation-induced electron-hole pairs is lost due to their recombination in the plasma column created by the high LET particle track $[17,18,19]$. This recombination loss is reduced by the 
higher field which separates the charges in the plasma more rapidly. The highest reverse-bias applied to the conventional $p-i-n$ or Schottky a-Si:H diodes is limited by micro-plasma breakdown at the surface. The buried $p-i-n$ structure shown in Fig.3(a) suppresses this breakdown by moving the maximum field region away from the surface. For $5.7 \mu \mathrm{m}$ thick devices, we could raise the bias to about $50 \mathrm{~V} / \mu \mathrm{m}$ compared to about $20 \mathrm{~V} / \mu \mathrm{m}$ for the conventional $5 \mu \mathrm{m}, \mathrm{i}-\mathrm{i}-\mathrm{n}$ devices. This buried $\mathrm{p}-\mathrm{i}-\mathrm{n}$ structure should also help single particle detection of lower LET particles by the shorter charge collection time achieved in the higher fields. Higher count-rate performance and, in some case larger signal sizes will be obtained by the faster charge collection.

\section{Scintillation faceplate}

A scintillation faceplate [20] as shown schematically in Fig.3(b) is available from several manufactures [21]. Glass fibers containing rare-earth elements are fused and drawn to make a few $\mu \mathrm{m}$ columnar structure. Scintillation light is confined in each fiber thus the degradation of spatial resolution caused by the light spread is avoided in the indirect detection scheme shown in Fig.1(b). Although the light yield of the rare-earth element scintillation faceplates is not high enough to detect single minimum ionizing particles, it is adequate for detecting fluences in the current-mode operation.

The well-known inorganic scintillator CsI is promising for single particle detection. It has been shown that CsI(Tl) gives more light than $\mathrm{NaI}(\mathrm{Tl})$ [22]. Csl(Na) light yield is comparable to NaI(Tl). Properly made pure Csl with iodine vacancies has a $10 \mathrm{nsec}$ fast component and a relatively high light yield [23]. This fast component becomes important for applications which require fast detector response or a rigorous timing adjustment. CsI is made by several methods including vacuum evaporation. Vacuum evaporated CSI(Na) forms columns parallel to the evaporation direction. The column diameter can be controlled by heat treatment [24]. These columns form natural light pipes that function similarly to the optical fiber described earlier, confining the light to produce a sharper image at the phospher-detector interface. Csl(Na) layers $300 \mu \mathrm{m}$ thick with $50 \mu \mathrm{m}$ column diameter are routinely made by manufactures of $\mathrm{X}$-ray image intensifiers [25]. Thicker Csl faceplate with a larger columnar diameter can be made by melting and recrystallizing Csl inside a Honeycomb structure. Preliminary results at our laboratory show both of these methods to be promising. 
Interdigitated electrode

For the indirect detection scheme shown in Fig.1(b), the a-Si:H layer thickness of $1-2 \mu \mathrm{m}$ is enough to absorb all the scintillation light from a phosphor. For single particle detection, the delta noise arising from the large detector capacitance must be reduced. The interdigitated electrode shown in Fig.3(c) provides a small capacitance at the expense of the response time or the need for a higher bias. The field non-uniformity inside a-Si:H layers with these types of electrodes may have interesting implication for new device struatures.

\section{High energy physics applications}

A number of secondary charged particles are emitted following a collision event of primary particles in high energy physics experiments. These particles are detected by position sensitive a$\mathrm{Si}: \mathrm{H}$ radiation detectors surrounding the collision site as shown schematically in Fig.4(a). Tracks of each particle can be identified by processing information from each detector. We have demonstrated the detectability of minimum ionizing particles by a-Si:H layers[5]. Thick a-Si:H layers formed into small pixels are essential to provide high signal to noise $(\mathrm{S} / \mathrm{N})$ ratio for direct detection of these particles. We have estimated the $S / N$ ratio of the pixel device for some assumed parameters[7]. Noise contributions from the detector capacitance, leakage current and the carrier trapping and emission in a-Si:H layer are shown to be low enough to provide high $S / N$ ratio for the direct detection of the minimum ionization events. An alternative is the indirect detection scheme shown in Fig.l(b). Even with the larger signal from indirect detection, reduction of the detector capacitance is essential if a thin a-Si:H layer is to be used. If a $300 \mu \mathrm{m}$ thick CsI(Tl) is coupled to the a-Si:H photodiode of $70 \%$ quantum efficiency, a signal size of 5000 electrons is expected. Due to the large area and high radiation resistivity required for this tracking device, despite smaller signal, a-Si:H radiation detectors may have advantages over the crystalline devices currently under development [26].

Another application in high energy physics is for calorimeters as shown schematically in Fig.4(b), which consist of multiple layers of converter material such as $\mathrm{Pb}$ and $\mathrm{U}$ and pixel detector layers. $\mathrm{A}$ high energy $\gamma$ ray produces electro-magnetic showers which consist of electrons and positrons. By recording the number of tracks and positions of the shower tracks, we can determine the energy and the 
direction of the incident $\gamma$ ray. Gas-filled detectors or crystalline $\mathrm{Si}$ detectors [27], currently used for recording the showers, could be replaced by a-Si:H detectors.

\section{Medical imaging applications}

There are many radiation detection devices used in medical imaging ranging from simple ones such as $X$-ray film, to elaborate systems such as a Positron Emission Tomograph (PET). We discuss the use of a-Si:H detectors in medical imaging by dividing these existing devices into two groups i.e. flux imaging devices and single photon imaging devices.

\section{Flux imaging devices}

$X$-ray film has been widely used due to its excellent spatial resolution despite the limited dynamic range, lack of real-time capability, inability for repeated use and need for additional efforts to obtain digitized images. The imaging plate developed by Fuji [28] solves most of the deficiencies of film except for time resolution. It requires a separate readout system to "develop" an image. An X-ray image intensifier is a real-time device and is often used in $X$-ray digital imaging[29]. The detecior part of a digital subtraction system consists of an image intensifier and a TV camera for image digitization. Since an image intensifier is a vacuum tube, it is bulky and mechanically delicate.

A pixel detector combined, with a scintillation faceplate as shown in Fig.5(a) could be used in place of these devices. A simple calculation below shows what the expected signal size is large enough to simplify the requirements for the readout electronics. Consider a typical $X$-ray exposure of 0.1 Roentgen as normally used for chest $X$ ray imaging. This translates to $3 \times 10^{9} 50 \mathrm{keV} X$-ray photons $/ \mathrm{cm}^{2}$. About $80 \%$ of these $\mathrm{X}$-ray photons are absorbed by $300 \mu \mathrm{m}$-thick CsI(Tl). From the known light yield of 50000 visible photons/1 $\mathrm{MeV}$ absorbed for CsI(TI), the $0.1 \mathrm{R}$ exposure produces $6 \times 10^{12}$ photons $/ \mathrm{cm}^{2}$ in $\mathrm{CsI}(\mathrm{Tl})$. As a conservative estimate, assume $50 \%$ light collection and $60 \%$ light to electron-hole pair conversion efficiency (quantum efficiency) of the a-Si:H photodiode. If we have $10^{4}$ pixels $/ \mathrm{cm}^{2}(100 \mu \mathrm{m} \times 100 \mu \mathrm{m}$ pixel size), each pixel produces $1.8 \times 10^{8}$ electron-hole pairs. The capacitance of a $1 \mu \mathrm{m}$ thick $100 \mu \mathrm{m} \times 100 \mu \mathrm{m}$ pixel is about $1 \mathrm{pF}$. Therefore, the average voltage signal on each pixel after $0.1 \mathrm{R}$ exposure is about $30 \mathrm{~V}$. The spatial resolution is determined by the pixel size and the scintillation light 
spread in the phosphor. The output image is inherently digitized and well-suited for sophisticated digital image processing techniques.

An X-ray Computed Tomograph (CT) is a more elaborate high flux imaging system[30], but the detector itself is not necessarily more sophisticated. A schematic drawing of an X-ray CT is shown in Fig.5(b). An X-ray-tube emitting a fan beam of $X$-rays is rotated around the body together with a one dimensional position sensitive $\mathrm{X}$-ray detector (1D-PSD). The transmission data through the patient's body known as projection profiles are stored in the computer for each X-ray-tube position and are used to reconstruct the transverse cross-sectional image of the $X$-ray attenuation coefficient distribution. An array of Xe-filled ionization chambers is normally used as the ID-PSD. Due to the geometrical magnification, the spatial resolution of this detector can be as low as a few $\mathrm{mm}$. A strip detector as shown in Fig.2(a) combined with a phosphor should work as well as the Xe chamber. Again, no elaborate readout amplifier is needed since it is a flux detector.

\section{Single photon imaging devices}

In nuclear medicine, radiopharmaceuticals are introduced into the body and $\gamma$ rays emitted from the body are detected to give an image of the radionuclide distribution[31]. Here, it is necessary to count individual $\gamma$ rays, unlike the flux detectors where the charges created by a number of $X$-rays are accumulated during the duration of the X-ray pulse. The scintillation camera is an example of this type of imaging device. It consists of a slab of $\mathrm{NaI}(\mathrm{Tl})$ coupled to a number of photomultiplier tubes (PMTs). Centroid calculation of the PMT outputs gives the position information of the incident $\gamma$ ray and the sum of all the PMT outputs gives the energy of the $\gamma$ ray. A mechanical collimator is placed in front of the scintillator to accept only the desired $\gamma$ rays to make an image of the radionuclide distribution in the body projected on the plane defined by the scintillator slab. A pixel device combined with a high light yield scintillator faceplate as shown schematically in Fig.5(a) can replace this scintillation camera. Here, the scintillator must be carefully chosen and $\mathrm{Csl}(\mathrm{Tl})$ appears promising because of its high light yield, high $\mathrm{Z}$ number and its built-in columnar structure. The thickness of CsI(Tl) should be more than $5 \mathrm{~mm}$ to ensure good detection efficiency for the most-commonly used $140 \mathrm{keV} \gamma$ rays from the Tc-99m compounds. The intrinsic spatial resolution of conventional scintillation cameras is more than $3 \mathrm{~mm}$. A pixel device shown in Fig.5(a) can have much higher spatial resolution if required. Since it 
doesn't have PMTs, it is compact, light weight, and would need less routine adjustments than the conventional PMT array.

Single photon emission computed tomographs (SPECT) and positron emission tomographs (PET) are newer devices in nuclear medicine. Schematic drawings are shown in Fig.6. Projection lines are defined by the detector position and the direction of the collimator holes in SPECT or by the two detector positions giving outputs simultaneously due to detection of the two $511 \mathrm{keV} \gamma$ rays created by a positron annihilation in a PET. These projection lines are stored in the computer and the same mathematical process with a X-ray CT gives the transverse cross-sectional image of the radionuclide distribution in the body. The SPECT or PET cameras of the "multicrystal type" employ a number of small scintillator+PMT combinations placed on a circular array to form a detector ring. We can replace these scintillation detectors by a scintillator array combined with an a-Si:H photodiode array as shown in Fig.6(a) and (b). This a-Si:H array is an 1D-PSD with spatial resolution of a few $\mathrm{mm}$. Another type of SPECT or PET is the polygonal array of scintillation cameras. As discussed earlier, a scintillation camera can be made of a scintillation faceplate and an a-Si:H pixel device. These can be mounted in a polygonal array as shown in Fig.6(c) and (d). Some care must be taken in reconstructing an image from the projection profiles taken by the polygonal array of scintillation cameras due to the insensitive gaps between the adjacent cameras [32]. A polygonal type camera has the advantage of a better coverage of the body. Extra attention must be paid in selecting a scintillation material for a PET, namely. the decay time of scintillation must be short to reduce false projection lines caused by accidental coincidences under high countrate situations. The usual choices to date are BGO or Nal(Tl) crystals. Unfortunately, BGO gives only a small amount of light ( 8000 photons/1MeV absorbed)[21] and scintillation light detection by a position sensitive photodiode is not an easy task [33]. We estimate that pure CsI with iodine vacancies is promising because it has a fast component $(<20 \mathrm{nsec})$ and a relatively high light yield $(17000$ photens $/ 1 \mathrm{MeV})$. When all of the light is confined to the narrow path of the honeycomb structure, the photodiode will give an adequately large signal.

Determination of the $\gamma$ ray direction is achieved electronically in a PET and mechanically in a scintillation camera and SPECT, Electronic collimation is possible for the latter case, too[34]. The principle of this collimation is illustrated in Fig.7. Those $\gamma$ rays that undergo Compton scattering in Detector 1 and Photoelectric event of 
the resultant scattered $\gamma$ rays in Detector 2 are recorded. The angle of a cone on which the direction of the incident $\gamma$ ray lies is determined from the energy of the scattered electron measured by Detector 1. The generating line of the cone is a line connecting, the interaction points in Detector 1 and 2. Therefore, the spatial resolution of this system is determined by the energy resolution of Detector 1 and the spatial resolutions of Detectors 1 and 2. Various types of radiation detectors have been proposed as the candidates for the two detectors in the electronically collimated system $[35,36]$. The original combination in [35] is a multi-element $\mathrm{Ge}$ detector plus a scintillation camera whose spatial resolution is not better than a few $\mathrm{mm}$ in both cases. An a-Si:H photodiode array combined with a gas scintillation detector is a possible candidate for Detector 1 since a gus scintillation detector has a large area and an excellent energy resolution and an a-Si:H photodiode array is made to have good spatial resolution. An a-Si:H photodiode array combined with a scintillation faceplate is a candidate for Detector 2 due to its excellent spatial resolution.

\section{Material science and life science applications}

Radiation detectors are used in material science for recording diffr:ction patterns in $X$-ray crystallography and $X$-ray or synchrotron radiation transmission images in non-destructive studies of materials. Bateman gives a review of the existing devices used for these studies [37]. As pointed out there, the principal restriction on the wide-spread use of semiconductor detectors is their limited area. A-Si:H may be a better choice for the detector material for such applications.

In $\mathrm{X}$-ray crystallography, monoenergetic characteristic $\mathrm{X}$-rays such as the $\mathrm{Cu} \alpha$-line (8keV) are detected by curved or flat 1D-PSD such as film or multiwire proportic nal chambers(MWPC) [38,39]. ASi:H alloys on flexible sibstrates can be used in place of fiim or MWPC detectors. Since the diffracted X-ray flux intensity is low for these studies, single photon detection is preferred over flux integration. The pulse mode operation (counting a single $X$-ray photon) of the indirect detection scheme is somewhat difficult because, for example, one $8 \mathrm{keV} X$-ray photon creates only about 400 photons in CsI(TI). Direct detection of a single $8 \mathrm{keV} \mathrm{X}$-ray photon needs careful design of the readout electronics because the expected number of electron-hole pairs is around 1300. Table 1 summarizes the probability of photoelectric effect of $8 \mathrm{keV}$ and $17 \mathrm{keV} X$-ray 
photons in three kinds of a-Si:H alloys i.e. $100 \% \mathrm{Si}, 90 \% \mathrm{Si}+10 \% \mathrm{Ge}$, $80 \% \mathrm{Si}+20 \% \mathrm{Ge}$. A-Si:Ge:H alloys with good quality can be made with the Ge content up to $20 \%$. Layer thicknesses of $30-50 \mu \mathrm{m}$ provides similar detection efficiency as the pressurized Xe-filled MWPCs.

Table 1. Detection efficiency of a-Si:Ge:H alloys

\begin{tabular}{|c|l|l|l|l|l|l|}
\hline & \multicolumn{2}{|c|}{$100 \% \mathrm{Si}$} & \multicolumn{2}{c|}{$90 \% \mathrm{Si}+10 \% \mathrm{Ge}$} & $80 \% \mathrm{Si}+20 \% \mathrm{Ge}$ \\
\hline X-ray energy & $30 \mu \mathrm{m}$ & $50 \mu \mathrm{m}$ & $30 \mu \mathrm{m}$ & $50 \mu \mathrm{m}$ & $30 \mu \mathrm{m}$ & $50 \mu \mathrm{m}$ \\
\hline $8 \mathrm{keV}$ & 0.37 & 0.53 & 0.45 & 0.63 & 0.53 & 0.72 \\
\hline $17 \mathrm{keV}$ & 0.051 & 0.083 & 0.18 & 0.28 & 0.31 & 0.46 \\
\hline
\end{tabular}

In non-destructive studies of materials, where a flux of higher energy $X$-rays is employed, either direct or indirect detection of these $\mathrm{X}$-rays is possible by the $\mathrm{a}-\mathrm{Si}: \mathrm{H}$ detiotors operated by the integral mode (flux counting).

Even for $X$-ray microscopy [40] where the size advantage of a$\mathrm{Si} H$ is less significant, its high radiation resistivity is a desirable feature for these devices. When a large geometrical magnificarion is employed by making the ratio of the $X$-ray source-object distance to the object-detector distance sniall, a large detector area such as $10 \times 10 \mathrm{~cm}^{2}$ becomes important agjain.

In biology, : Atoradiography is a powerful tool for information on protein distributions and DNA sequences from radioisotopslabelled plates of organic bodies and electrophoresis gels Radioisotopes commonly used for these studies are $\mathrm{H}-3, \mathrm{C}-14$ and P32. These are all $\beta$-emitiers whose maximum energies are $18 \mathrm{keV}$, $155 \mathrm{keV}$ and $1.7 \mathrm{MeV}$, respectively. Positions and intensity distributions of these $\beta$-emitters are found by detecting the $\beta$ particles escaping from the plate or the gel with a $1 D$, or more recently 2D-PSD in close contact. $X$-ray film has been used widely. Multiwire proportional chambers (MWPC) have been applied for ID or 2D radiochromatography [41]. To ensure sufficient sensitivity, the thickness of the gas-filled detector is about $1 \mathrm{~cm}$, causing spatial resolution degradation due to the parallax error. Single $\beta$ particle detection for these radioisotopes can be done by a-Si:H radiation detectors either directly or indirectly with the exception of H-3. The detector thickness can be less than $1 \mathrm{~mm}$ and the sensitive area can be placed very close to tise plates or gels. H-3 detection requires careful design considerations on the readout electronics due to the small signal size. 


\section{Conclusion}

Thick a-Si:H layers with low ionizable defect density as well as thin a-Si:H layers combined with a phospher can detect various types of radiation encountered in various fields of science. Position sensitivity is obtained by dividing these layers into small strips or pixels and providing readout electronics for each strip or pixel. Electronics are designed either as the integral type for flux detection or the pulse type for single particle detection. The buried $p-i-n$ structure, high light yield scintillator CsI with column structure, and the interdigitated or mesh electrodes are all available to improve the detector performance. These a-Si:H radiation detectors will find their applications as tracking devices and calorimeters in high energy physics experiments, $X$-ray detectors for digital image processing such as a digital subtraction system and $X$-ray $C T$ in medical imaging, $\gamma$ ray detectors such as a scintillation camera, SPECT and PET in nuclear medicine. In material science and life science, thay can be applied to $X$-ray crystallography, non-destructive testing of materials, $X$-ray microscopy and radiochromatography. Each application imposes different requirements on the radiation detector systems. Whether a-Si:H detectors can replace existing devices such as film, gas-filled detectors, scintillation detectors and crystalline semiconductor detectors strongly depends on each application and it must be judged case by case. However in general, we expect the following advantages of the a-Si:H detectors over the existing devices; (a)larger area size, higher radiation resistance and lower cost than crystalline semiconductor detectors, (b)higher spatial resolution and detection efficiency than gas-filled detectors and (c)they are better suited for medical digital radiography than film or image intensifiers.

\section{Acknowledgements}

We would like to express our appreciation to C.C.Tsai of Xerox PARC and J.Xi of Glasstech Solar for making a-Si:H samples. We are also grateful to T.Gee and A.Lyon of LBL for phosphor preparation. This work was supported by the Director, Office of Energy Research, Office of High Energy and Nuclear Physics, Division of High Energy Physics of the U.S. Department of Energy under contract No. DEAC03-76SF00098. 


\section{References}

1. Wei Guang-Pu, H.Okamoto and Y.Hamakawa, "AmorphousSilicon Photovoltaic X-Ray Sensor," Jpn. J. Appl. Phys. Vol.24(1985)1105.

2. K.Mochiki, K.Hasegawa and S.Namatame, "Amorphous Silicon Position-Sensitive Detector," Nucl. Instr. and Meth. A273 (1988)640.

3. Y.Naruse and T.Hatayama, "Metal/Amorphous Silicon Multilayer Radiation Detectors," IEEE Trans. Nucl. Sci. NS-36 (1989)1347.

4. F.Gouldings and D.Landis, "Signal Processing for Semiconductor Detectors," IEEE Trans. Nucl. Sci. NS-29(1982) 1125.

5. S.N.Kaplan, I.Fujieda, V.Perez-Mendez, S.Qureshi, W.Ward and R.A.Street, "Detection of Minimum-Ionizing Particles in Hydrogenated Amorphous Silicon," Nucl. Instr. and Meth. A273 (1988)611.

6. I.Fujieda, G.Cho, S.N.Kaplan, V.Perez-Mendez, S.Qureshi, W.Ward and R.A.Street, "Detection of Charged Particles in Thick Hydrogenated Amorphous Silicon Layers," Proc. Mater. Res. Soc. Vol.118(1988)469.

7. V.Perez-Mendez, S.N.Kaplan, G.Cho, I.Fujieda, S.Qureshi, W.Ward and R.A.Street, "Hydrogenated Amorphous Silicon Pixel Detectors for Minimum Ionizing Particles," Nucl. Instr. and Meth. A273(1988)127.

8. S.Qureshi, V.Perez-Mendez, S.N.Kaplan, I.Fujieda, G.Cho and R.A.! Street, "Signal Generation in a Hydrogenated Amorphous Silicon Detector," IEEE Trans. Nucl. Sci. NS-36(1989)194.

9. V.Perez-Mendez, G.Cho, I.Fujieda, S.N.Kaplan, S.Qureshi and R.A. Street, "The Application of Thick Hydrogensited Amorphous Silicon Layers to Charged Particle and X-Ray Detection," LBL26998, Presented at Mater. Res. Soc. Spring Meeting, San Diego April 25-28, 1989.

10. H.Curtins, W.Wyrsch, and A.V.Shah, "High-Rate Deposition of Amorphous Hydrogenated Silicon: Effect of Plasma Excitation Frequency," Electron. Lett.23(1987)228.

11. S.Oda, J.Noda and M.Matsumura, "Preparation of a-Si:H Films by VHF Plasma CVD," Proc. Mater. Soc.Vol.118(1988) 117.

12. H.Chatham and P.K.Bhat, "High Deposition Rate P-I-N Solar Cells Prepared from Disilane Using VHF Discharges," Presented at Mater. Res. Soc. Spring Meeting, San Diego April 25-28, 1989.

13. M.Ohnishi, H.Nishiwaki, K.Uchihashi, K.Yoshida, M.Tanaka, K.Ninomiya, M.Noshikuni, N.Nakamura, S.Tsuda, S.Nakano, T.Yazaki and Y.Kuwano, "Preparation and Properties of a-Si 
Films Deposited at a High Deposition Rate under a Magnetic Field," Jpn. J. Appl. Phys. Vol.27(1988)40.

14. S.A.Kleinfelder, W.C.Carithers, R.P.Ely, C.Haber, F.Kirsten and H.G.Spieler, "A Flexible 128 Channel Silicon Strip Detector Instrumentation Integrated Circuit with Sparse Data Readout," IEEE Trans. Nucl. Sci. NS-35(1988)171.

15. B.Equer and A.Karar, "Amorphous Semiconductors for Particle Detection: Physical and Technical Limits and Possibilities," Nucl. Instr, and Meth. A275(1989)558.

16. V.Perez-Mendez, G.Cho, I.Fujieda, S.N.Kaplan, S.Qureshi and R.A. Street, "Proposed Thin Film Electronics for a-Si:H PIXEL Detectors," LBL-26254.

17. V.Perez-Mendez, S.N.Kaplan, W.Ward, S.Qureshi and R.A.Street, "Signal, Recombination Effects and Noise in Amorphous Silicon Detectors," Nucl. Instr. and Meth. A260(1987)195.

18. B.Equer and A.Karar, "Effects of Primary ionization in Amorphous Silicon Detectors," Nucl. Instr. and Meth. A271 (1988)574.

19. J.Dubeau, T.Pochet, A.Karar, L.A.Hamel, B.Equer, J.P.Martin, S.C. Gujrathi and A.Yelon, "Response of a-Si:H Detectors to Protons and Alphas," Proc. Mater. Soc. Vol.118(1988)439.

20. R.Ruchti, B.Baumbaugh, J.Bishop, N.Biswas, N.Cason, R.Erichsen, V.Kenney, A.Kreymer, R.Mountain and W.Shephard, "Scintillating Glass, Fiber-Optic Plate Detectors for Active Target and Tracking Applications in High Energy Physics Experiments," IEEE Trans. Nucl. Sci. NS-31(1984)69.

21. Synergistic Detector Designs, Collimated Hole.

22. I.Holl, E.Lorenz and G.Mageras, "A measurement of the Light

Yield of Some Common Inorganic Scintillators," IEEE Trans. Nucl. Sci. NS-35 (1988)105.

23. S.Kubota, S.Sakuragi, S.Hashimoto and J.Ruan, "A New

Scintillation Material: Pure CsI with 10ns Decay Time," Nucl. Instr. and Meth. A268(1988)275.

24. A.L.N.Stevens and A.D.M.Schrama de Paum, "Vapor-Deposited CsI:Na Layers, 1. Morphologic and Crystallographic Properties," Philips Res. Repts 29(1974)340.

25. M.Tsuda and Y.Kimura, "Background Generation at Input Screens of X-ray Image Intensifiers," Jpn. J. Appl. Phys. Vol.24(1985) 1087.

26. E.H.M.Heijne and P.Jarron, "Development of Silicon Pixel Detectors: an Introduction," Nucl. Instr. and Meth. A275(1989) 467. 
27. J.Bourotte, M.Haguenauer, E.Pare, V.Innocente, S.Lanzano,

K.Kasahara, Y.Muraki, T.Yuda, A.Nakamoto, H.Murakami, T.Doke, T.Kashiwagi, J.Kikuchi and K.Masuda, "Energy and Position Resolution of Silicon Calorimeter Used in the UA7 Experiment," Nucl. Instr. and Meth. A274(1989)129.

28. J.Miyahara, K.Takahashi, Y.Amemiya and N.Kamiya, "A New Type of X-Ray Area Detector Utilizing Laser Stimulated Luminescence," Nucl. Instr, and Meth. A246(1986)572.

29. H.Roehrig, S.Nudelman, H.D.Fisher, M.M.Frost and M.P.Capp, "Photoelectronic Imaging for Radiology," IEEE Trans. Nucl. Sci. NS-28(1981)190.

30. L.Axel, P.H.Arger and R.A.Zimmerman, "Applications of Computerized Tomography to Diagnostic Radiology," Proc. IEEE Vol.71 No.3 (1983)293.

31. T.F.Budinger, G.T.Gullberg and r.H.Huesman, "Emission Computed Tomography," Ch.5, Image Rocenstruction from Projections, Edited by G.T.Herman, Springer-Verlag Berlin Heidelberg 1979.

32. J.M.Ollinger and J.S.Karp, "An Evaluation of Three Algorithms for Reconstructing Images from Data with Missing Projections," IEEE Trans. Nucl. Sci. NS-35(1988) 629.

33. S.E.Derenzo, W.W.Moses, H.C.Jackson, B.T.Turko, J.L.Cahoon, A.B. Geyer and T.Vuletich, "Initial Characterization of a Position'Sensitive Photodiode/BGO Detector for PET," IEEE Trans. Nucl. Sci. NS-36(1989)1084.

34. M.Singh and D.Doria, "An Electronically Collimated Gamma Camera for single photon emission tomography. Part 1: Theoretical Considerations and Design Criteria," Med. Phys. 10(1983)421.

35. I.Fujieda and V.Perez-Mendez, "Theoretical Considerations of a New Electronically Collimated Gamma Camera Utilizing Gas Scintillation," Jpn. J. Appl. Phys. Vol. 25(1986)1925.

36. C.J.Solomon and R.J.Ott, "Gamma Ray Imaging with Silicon Detectors-A Compton Camera for Radionuclide Imaging in Medicine," Nucl. Instr. and Meth. A273(1988)787.

37. J.E.Bateman, "Detectors for Condensed Matter Studies," Nucl. Instr. and Meth. A273(1988)721.

38. V.Perez-Mendez, P.Wiedenbeck, C.N.Wagner and E.Woelfel, "Curved Anode Wire Chambers for X-ray Diffraction Applications," Nucl. Instr. and Meth. 228(1985)564.

39. Hamlin, Cork, Howard, Nielsen, Vernon, Matthews, PerezMendez, Xuong, "Characteristics of Flat Multiwire Area 
Detectors for Protein Crystallography," J. Appl. Crystallography 14(1981)85.

40. J.E.Bateman, G.R.Moss, P.Rocket and S Webb, "Digital X-Ray Microscopy with a Microfocal X-Ray Generator and an MWPC Area Detector," Nucl. Instr. and Meth. A273(1988)767.

41. C.W.E. Van Eijk, R.W.Hollander and S.M.Van der Sluis, "An MWPC for Beta Radiochromatography," Nucl. Instr. and Meth. A273(1988)764. 


\section{Figure Captions}

Fig.1 Detection principles of a-Si:H radiation detectors.

(a) Direct detection scheme. Incoming radiation creates a large number of electron-hole pairs in the depleted intrinsic region. These charges are displaced by the field and the induced charges on the electrodes are amplified by the readout electronics.

(b) Indirect detection scheme. Incoming radiation creates a large number of visible light photons in a phospher. These visible photons enter the a-Si:H photodiode.

(c) Calorimeter scheme. Incoming radiation creates energetic secondary particles in the converter material such as heavy metals. Those escaping from the converter layers are detected by the a-Si:H layers directly.

Fig.2 Schematics of position sensitive detectors (PSDs).

(a) One dimensional PSD (strip detector). The top electrode is divided into narrow strips and each strip is wire-bonded to the separate amplifier IC chip to take advantage of the existing crystalline $\mathrm{Si}$ readout electronics.

(b) One dimensional PSD with the built-in readout electronics made of a-Si:H or poly-Si thin film transistors (TFTs).

(c) Two dimensional PSD (pixel detector) with the built-in readout electronics made of a-Si:H or poly-Si TFTs.

Fig.3 Promising technologies.

(a) The buried pin structure. By including lightly doped layers, the electric field is tailored so that the maximum field occurs inside the material thus avoiding the micro-plasma breakdown at surfaces.

(b) Scintillation faceplate. Scintillation light is confined in a narrow region parallel to the plane normal. This columnar structure is made by a proper heat treatment for the evaporated CsI. Or scintillation glass tubing containing a rareearth element is stacked and drawn to form a scintillation faceplate.

(c) Interdigitated or mesh electrode to reduce the detector capacitance.

Fig.4 Applications in high energy physics experiments. 
(a) Tracking device. Particle tracks are identified by processing the information from the PSDs surrounding the colliding beam interaction point.

(b) Calorimeter. Many layers of a converter material and an aSi:H detector are stacked together. A high energy $\gamma$ ray produces an electro-magnetic shower. The number of tracks and positions determine the energy and the direction of the incident $\gamma$ ray.

Fig.5 Applications in medical imaging. Part 1. (a)2D-PSD. A 2D a-Si:H photodiode array combined with either a $300 \mu \mathrm{m} \mathrm{CsI(Tl)} \mathrm{with} \mathrm{fine} \mathrm{columnar} \mathrm{structure} \mathrm{or} \mathrm{a} \mathrm{scintillation}$ faceplate containing a rare-earth element can be used for recording Xray transmission images. A single $\gamma$ ray imaging becomes possible when a $5 \mathrm{~mm}$ thick CsI(Tl)-filled faceplate is combined with an a-Si:H photodiode equipped with a proper readout electronics.

(b) ID-PSD. A 1D a-Si:H photodiode array combined with a similar ID scintillator array can be used in place of the Xefilled ionization chamber currently employed in an Xray CT.

Fig.6 Applications in medical imaging. Part 2.

Detectors for a SPECT and a PET can be built by the scintillation camera described in the text. Cylindrical and hexagonal configurations are shown for a SPECT ((a),(c)) and for a PET ((b),(d)).

Fig.7 Electronically collimated system. The scintillation camera described in the text can be used as Detector 2 and a 2D a-Si:H photodiode array can be used as a part of Detector1. For detail, see the text. 
18

Radiation

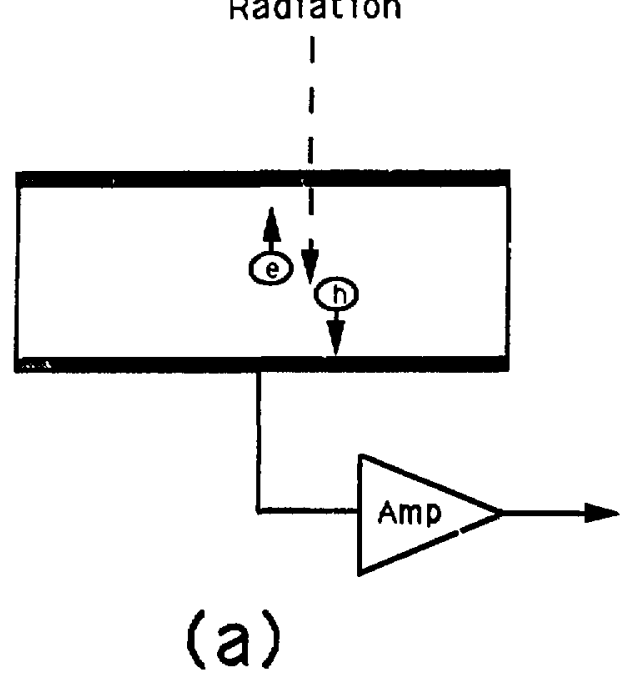

Radiation

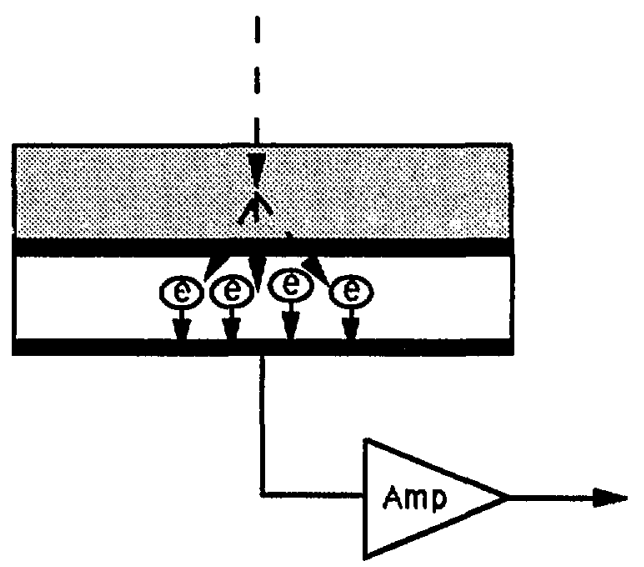

(b)

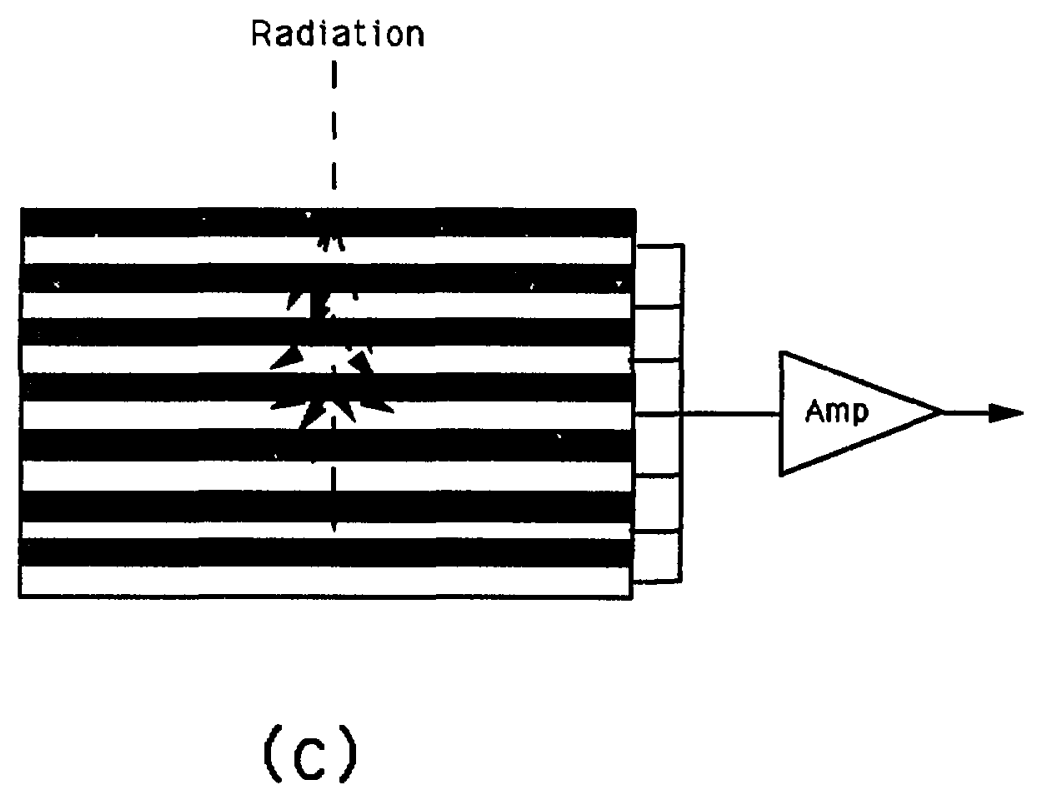

Fig. 1 
(a)

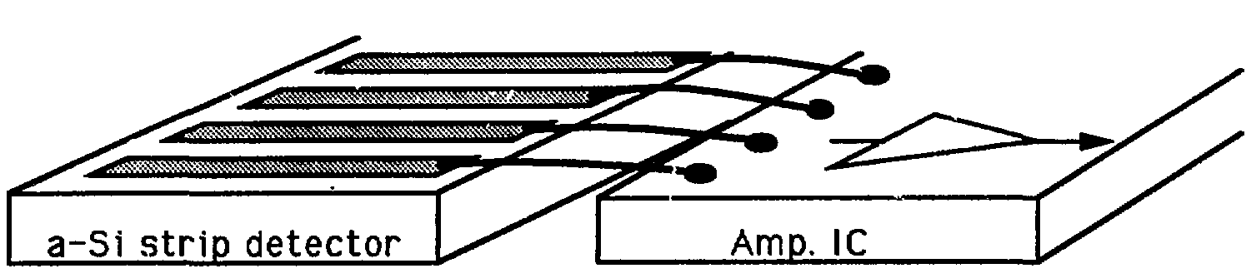

(b)

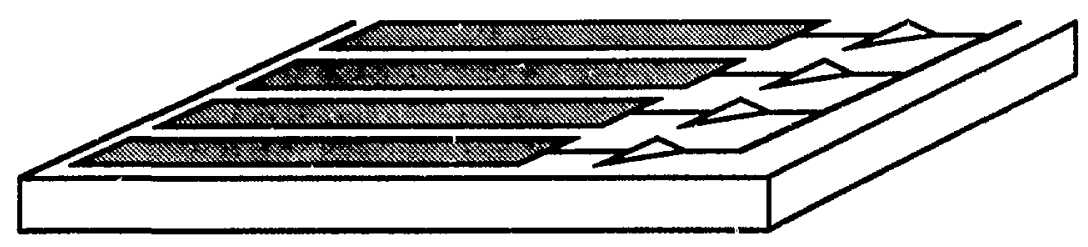

(c)

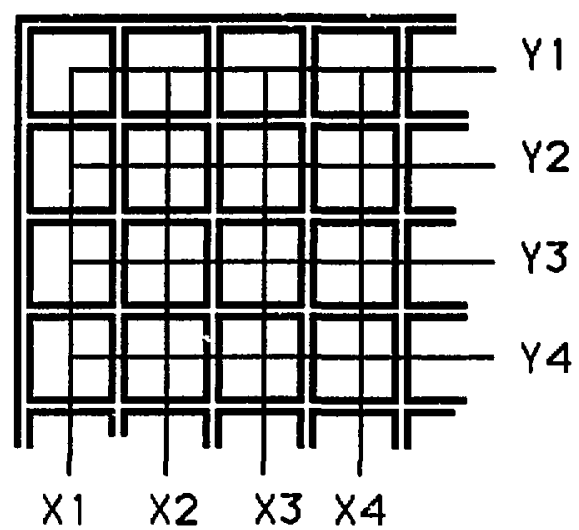

PIXEL

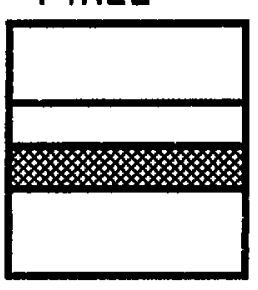

$\left.\begin{array}{l}\text { Detector } \\ \text { Readout Bus } \\ \text { Electronics }\end{array}\right]$

Substrate

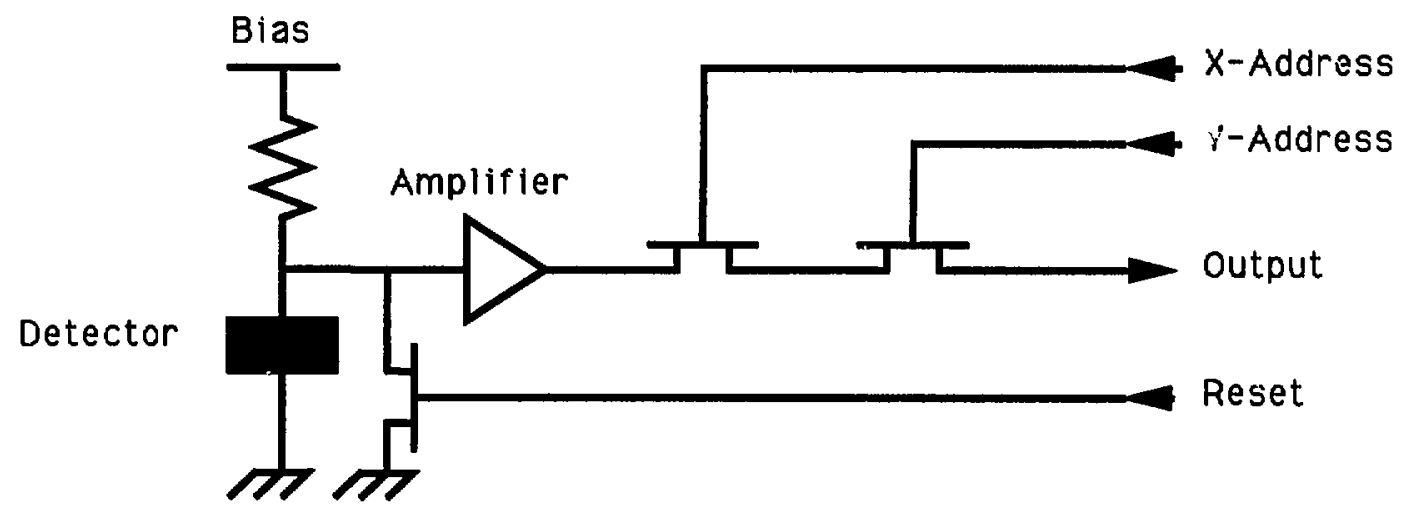

Fig. 2 

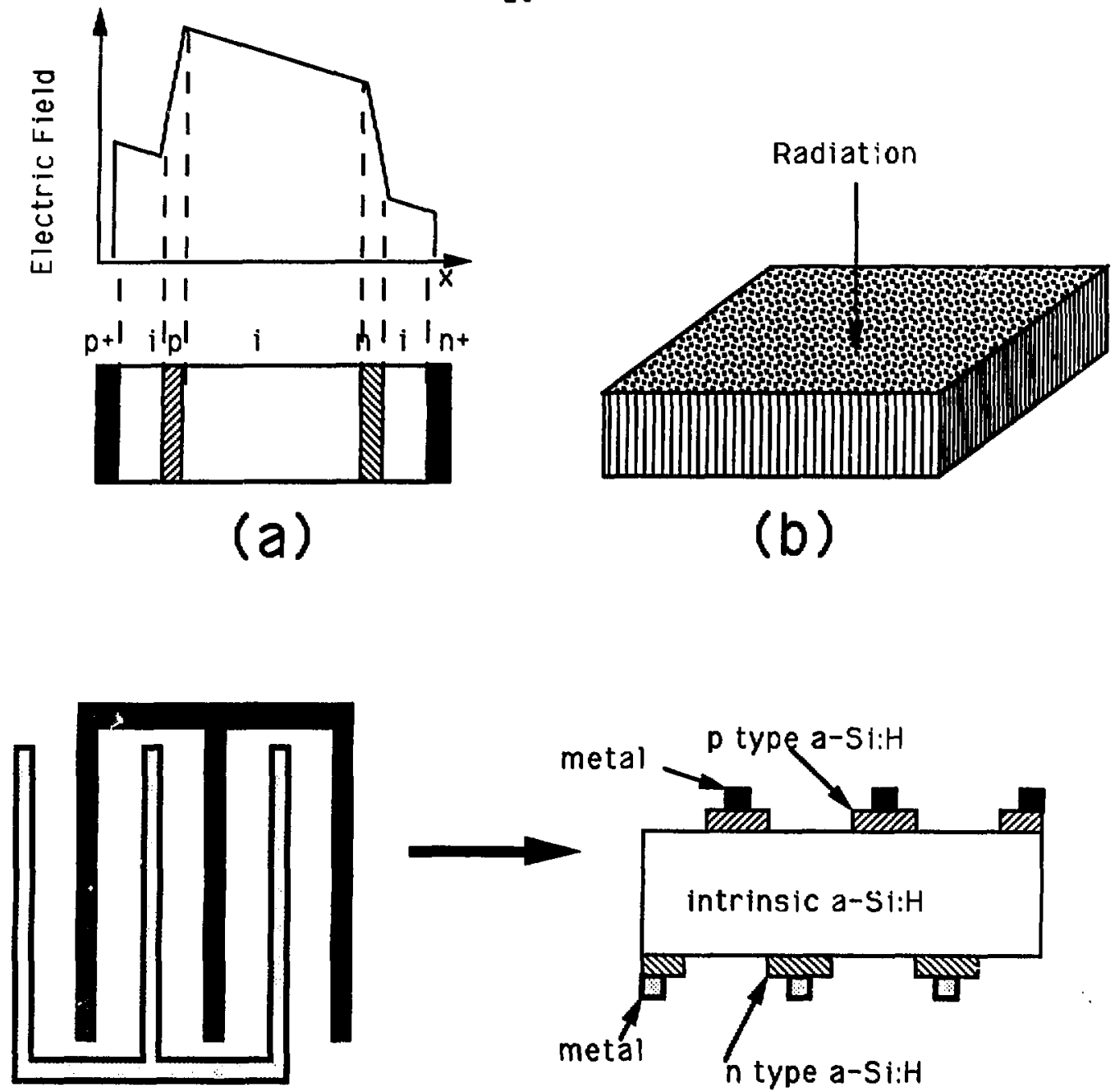

(c)

Fig. 3 
Colliding Beam

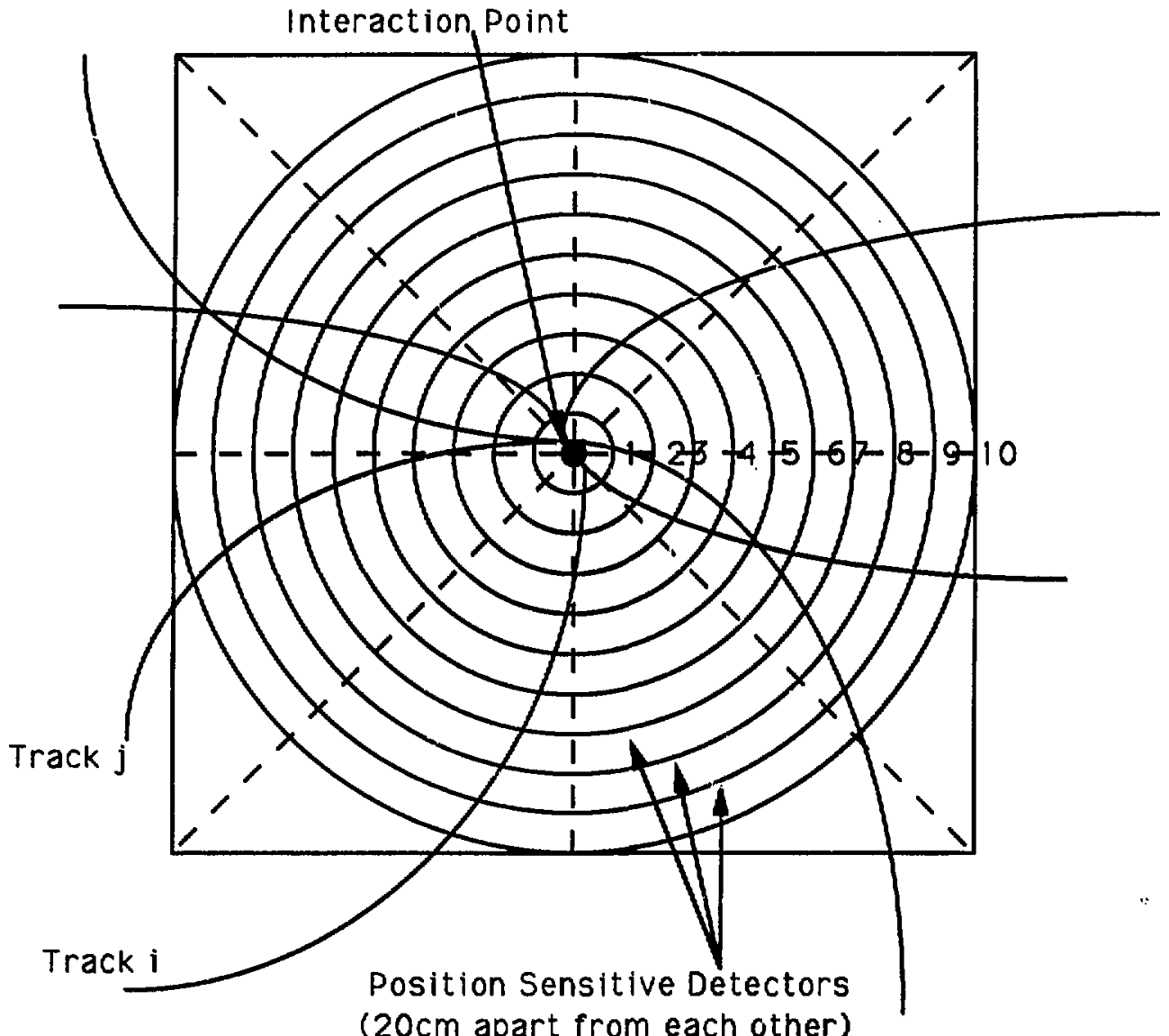

(a)

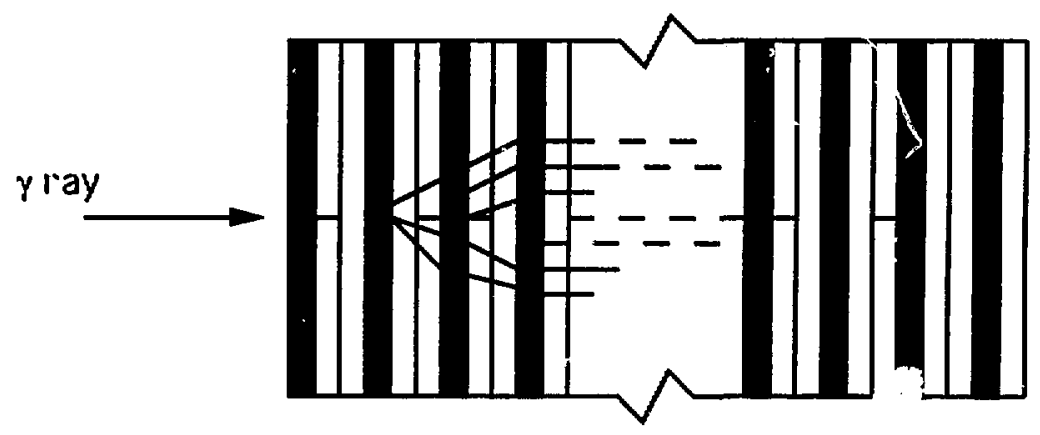

(b)

Fig. 4 


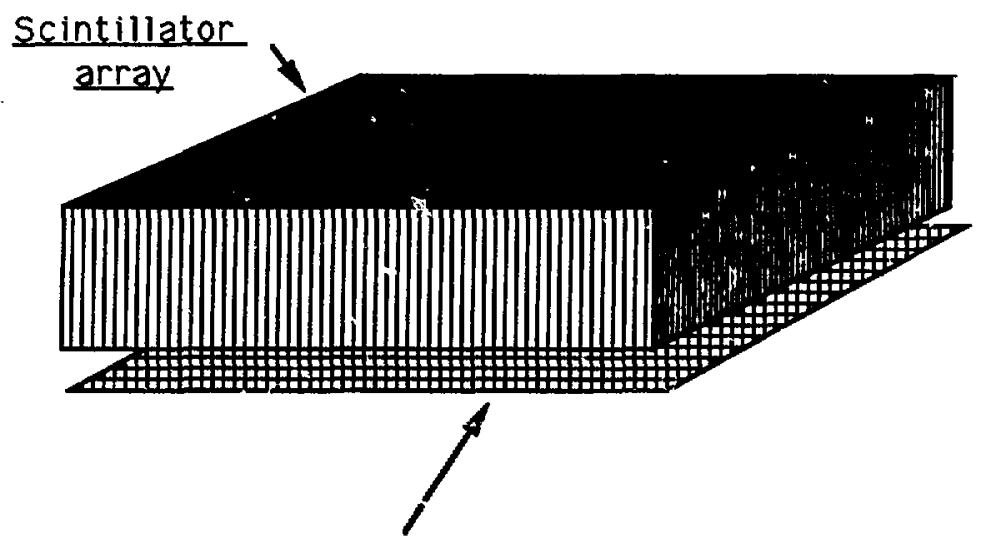

a-S120 Photodiode array

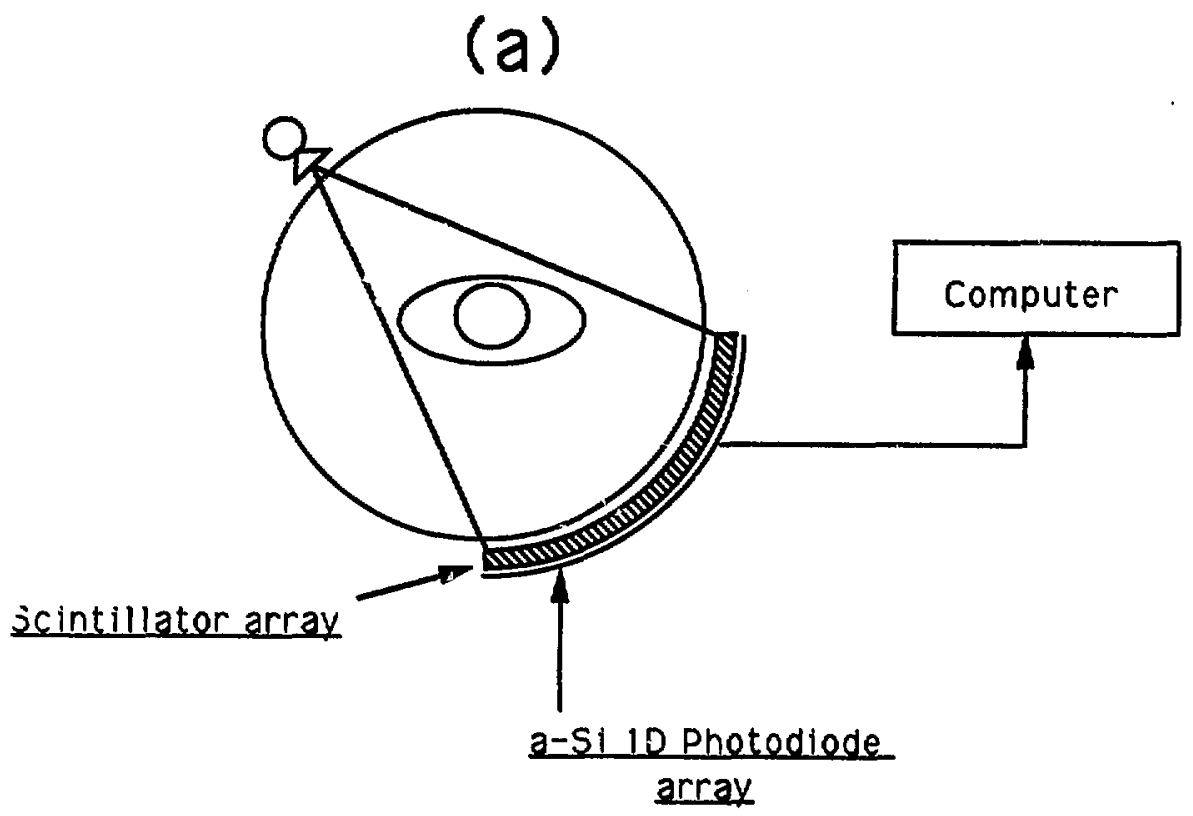

(b)

Fig. 5 

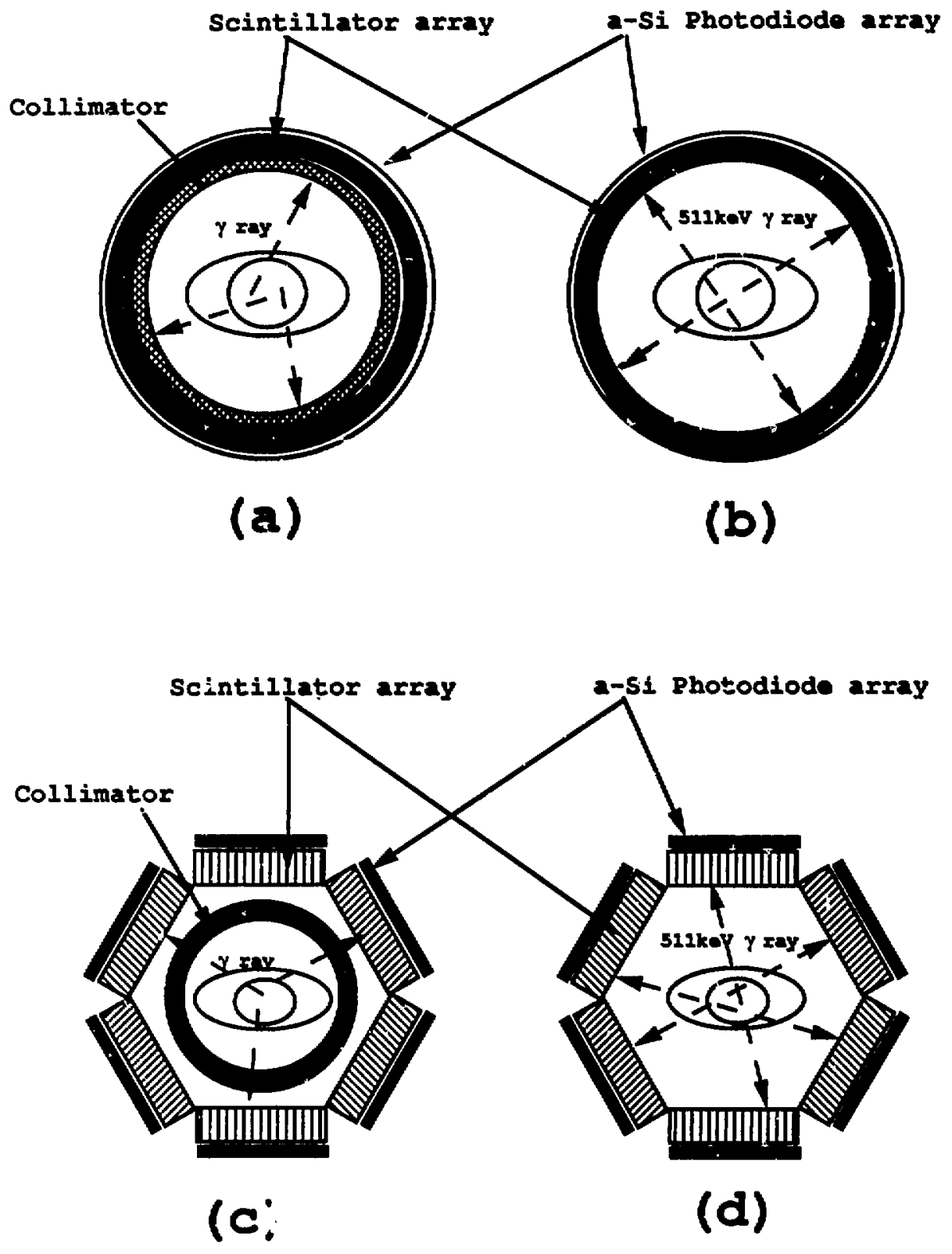

Fig. 6 


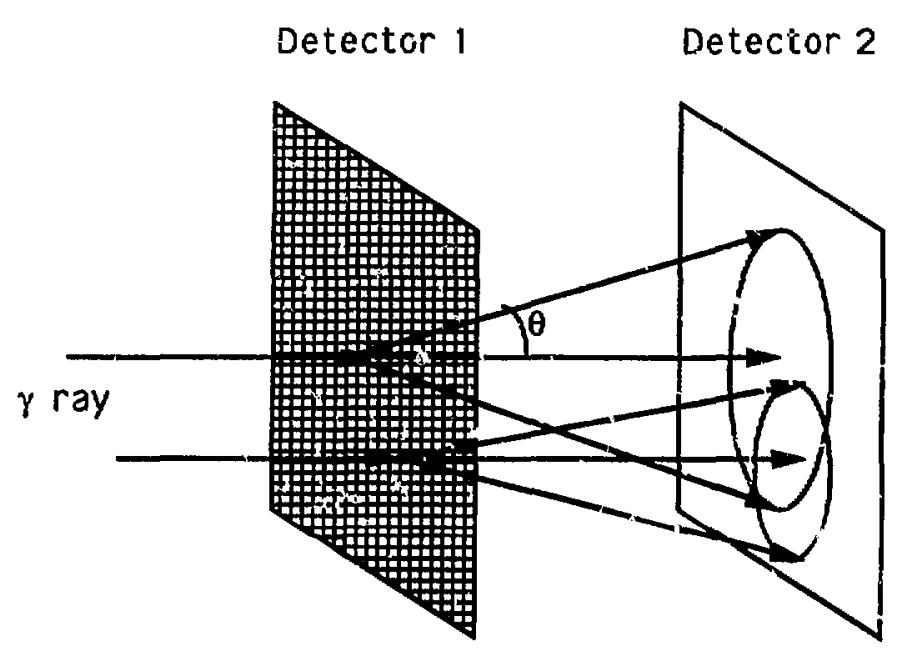

Fig. 7 